\title{
Thyroglobulin Recovery Rate
}

National Cancer Institute

\section{Source}

National Cancer Institute. Thyroglobulin Recovery Rate. NCI Thesaurus. Code C147435.

The determination of the thyroglobulin recovery rate present in a sample. 